\title{
Manifestações Sistêmicas Adversas em Medicina Intensiva após Realização de Perfusão Isolada de Membro com Melfalan e Hipertermia. Relato de Caso*
}

\author{
Adverse Systemic Reactions in Intensive Care Medicine after Isolated \\ Limb Perfusion with Melphalan and Hyperthermia. Case Report
}

Fernando Oetterer Arruda1, Hélio Penna Guimarães², Luiz Fernando dos Reis Falcão³, Patrícia Helena Rocha Leal', Renato Delascio Lopes², Ivan Dunshee de Abranches Oliveira Santos ${ }^{4}$, José Luiz Gomes do Amaral5.

\section{RESUMO}

JUSTIFICATIVA E OBJETIVOS: A presença de efeitos adversos, inerentes a todos os tratamentos, justifica a necessidade do profundo conhecimento pela equipe médica para prevenção e tratamento de eventuais disfunções orgânicas, reduzindo o seu impacto. O objetivo deste estudo foi relatar um caso de paciente que apresentou diversas manifestações sistêmicas, após a realização de perfusão isolada de membro com melfalan e hipertermia.

RELATO DO CASO: Paciente do sexo feminino, 64 anos, branca, com diagnóstico de melanoma na região de maléolo medial do membro inferior esquerdo. Seis meses após a excisão da lesão, realizou-se per-

1. Médico Anestesiologista da Disciplina de Anestesiologia, Dor e Medicina Intensiva da UNIFESP - EPM.

2. Médico Assistente da UTI da Disciplina de Clínica Médica da UNIFESP - EPM; Título de Especialista em Terapia Intensiva pela AMIB-AMB.

3. Acadêmico de Medicina da UNIFESP - EPM.

4. Professor Adjunto da Disciplina de Cirurgia Plástica da UNIFESP - EPM.

5. Professor Titular da Disciplina de Anestesiologia, Dor e Medicina Intensiva da UNIFESP - EPM. Título de Especialista em Terapia Intensiva pela AMIB-AMB

*Recebido da Unidade de Terapia Intensiva da Disciplina de Clínica Médica da Universidade Federal de São Paulo, Escola Paulista de Medicina (UNIFESP-EPM), São Paulo, SP.

Apresentado em 05 de agosto de 2006

Aceito para publicação em 20 de novembro de 2006

Endereço para correspondência:

Dr. Fernando Oetterer Arruda

Rua Humberto I, 908/62 - Vila Mariana

04018-033 São Paulo, SP

Fone: (11) 9116-9260

E-mail: foarruda@uol.com.br

(C)Associação de Medicina Intensiva Brasileira, 2006 fusão isolada do membro, com melfalan e hipertermia para conter o processo em evolução de possível metástase. A admissão na UTI apresentou síndrome da resposta inflamatória sistêmica (SIRS) com instabilidade hemodinâmica refratária à expansão volêmica. Durante a internação evoluiu com quadro de edema agudo de pulmão e disfunção miocárdica, revertidos com sucesso, depois de adequada intervenção terapêutica.

CONCLUSÕES: A presença de efeitos adversos, inerentes a todos os tratamentos oncológicos, justifica a necessidade do conhecimento pela equipe da terapia intensiva para prevenção e tratamento de eventuais disfunções orgânicas, reduzindo o impacto de sua morbimortalidade.

Unitermos: melanoma, melfalan, reperfusão isolada, síndrome de reperfusão.

\section{SUMMARY}

BACKGROUND AND OBJECTIVES: The presence of adverse reactions, inherent to all treatments, justifies the necessity of deep knowledge, by the medical team of the prevention and treatment of occasional organic dysfunctions, reducing its impact. The purpose of this paper is to report a case comprising the several systemic adverse reactions after perfusion of limb with melphalan and hyperthermia.

CASE REPORT: A white female, 64-years old patient with diagnosis of melanoma in the medial malleoli region of the left lower limb. Six months after surgical removal of wound, an isolated perfusion of limb was carried out with melphalan and hyperthermia in order to curb the possible metastatic process in evolution. At admission in the ICU, the patient presented systemic inflammatory response syndrome (SIRS) with refractary 
hemodynamic instability to volemic expansion. During internation the patient evolved to acute lung edema and myocardial dysfunction, all reverted successfully.

CONCLUSIONS: The potential presence of adverse reactions, inherent to all treatments, justify the necessity of knowledge by the intensive care team in the prevention and treatment of occasional organic dysfunctions, reducing the impact of morbidity and mortality.

Key Words: isolated perfusion, melanoma, melphalan, reperfusion syndrome.

\section{INTRODUÇÃO}

O melanoma cutâneo é sem dúvida uma das formas mais prevalentes de neoplasia; estimou-se em 2004, que 55.000 americanos foram diagnosticados como portadores dessa doença e 7.900 evoluíram ao óbito, sendo mais comum em pacientes com idade entre 20 e 29 anos $^{1}$.

O uso de quimioterápicos, por via intra-arterial tem demonstrado bons resultados no tratamento de diversos tipos de tumores. Através do isolamento da perfusão local, com circuito de circulação extracorpórea, altas doses de fármacos podem ser administrados em associação com procedimentos para hipertermia local, com vantagens de potencializar a ação e limitar a sua toxicidade apenas ao local necessário². A morbidade e mortalidade associadas a esse procedimento são baixas, e, portanto, este vem sendo freqüente estratégia de escolha para o tratamento de neoplasias, quando são necessárias altas doses do fármaco, sem o desconforto de eventos adversos sistêmicos. As melhores respostas foram obtidas em casos de melanoma recorrente nos membros, com metástase para linfonodos, onde se obteve sensível redução do número de amputações e aumento da sobrevida. Além dos membros, a técnica de perfusão hipertérmica isolada já foi descrita para pelve, cabeça, pescoço e pele da mama ${ }^{2,3}$.

A maior parte das publicações apontam apenas para efeitos da infusão venosa sistêmica, pouco se relatando aos eventuais efeitos da perfusão regional isolada ${ }^{4-6}$.

A presença de efeitos adversos, inerentes a todos os tratamentos, justifica a necessidade do profundo conhecimento pela equipe clínica e de terapia intensiva na prevenção e tratamento de eventuais disfunções orgânicas, reduzindo o seu impacto.

O objetivo deste estudo foi relatar um caso de paciente que apresentou diversas manifestações sistêmicas após a realização de perfusão isolada de membro com melfalan e hipertermia.

\section{RELATO DO CASO}

Paciente do sexo feminino, 64 anos, branca, com diagnóstico de melanoma na região de maléolo medial do membro inferior esquerdo. A paciente não apresentava antecedentes mórbidos pessoais relevantes. Em fevereiro de 2004, realizou excisão da lesão, porém, após seis meses do procedimento foi indicada perfusão isolada do membro com melfalan e hipertermia para conter o processo de possível metástase em evolução.

O procedimento foi realizado com a paciente sob anestesia geral, com duração total de $5 \mathrm{~h} 45 \mathrm{~min}$. Foi utilizado melfalan $(1 \mathrm{mg} / \mathrm{kg})$, através de circuito de circulação extracorpórea parcial, sendo cateterizadas a artéria e a veia femoral.

Ao ser admitida na unidade de terapia intensiva (UTI) a paciente apresentava-se com intubação traqueal, pressão arterial de $70 \times 40 \mathrm{mmHg}$, FC 130 bpm em ritmo em três tempos com presença de $3^{a}$ bulha. Não haviam sido relatadas pela Anestesiologia intercorrências sugestivas de instabilidade hemodinâmica ou anafilaxia durante o procedimento. Realizou-se infusão de $2 \mathrm{~L}$ de cristalóide e $500 \mathrm{~mL}$ de colóide não protéico em $2 \mathrm{~h}$, com melhora da ausculta cardíaca, porém manutenção da hipotensão arterial e taquicardia, caracterizando, assim, instabilidade hemodinâmica refratária à expansão volêmica. Foi indicada instalação de noradrenalina, por via venosa, através de acesso venoso central. Adicionalmente, após a obtenção dos resultados dos exames de admissão, foram realizados procedimentos para correção de distúrbios hidroeletrolíticos de hipocalemia $(2,5 \mathrm{mEq} / \mathrm{L})$, hipocalcemia $(6,1 \mathrm{mg} / \mathrm{dL})$ e hipomagnesemia (1,3 mg/dL), anemia (hemoglobina 6,8 g/ $\mathrm{dL}$ prévia de $10 \mathrm{~g} / \mathrm{dL}$ ) e controle da saturação venosa central $\left(\mathrm{SvO}_{2}\right)$ através da análise de amostra colhida da veia cava superior por cateter central, que se mostrou abaixo dos limites toleráveis (66\%) de acordo com as rotinas da unidade $(70 \%$ a $75 \%)$. Não houve alteração dos valores basais dos demais exames, incluindo, CPK de $134 \mu / L$ (26-140) e CKMB de $16 \mu / L$ (até 25).

Após estabilização dos parâmetros hemodinâmicos, com a suspensão do fármaco vasopressor (noradrenalina), em aproximadamente $12 \mathrm{~h}$, a paciente foi retirada da ventilação mecânica e da prótese ventilatória, permanecendo estável pelas próximas $24 \mathrm{~h}$. Desenvolveu, então, quadro súbito de dispnéia, sudorese, agitação, presença de estertores subcrepitantes basais na ausculta pulmonar e concomitante aparecimento de imagem radiológica sugestiva de congestão pulmonar (Figura 1), confirmando o diagnóstico clínico de edema 
agudo de pulmão (EAP). Foi administrado diurético por via venosa e instalada ventilação mecânica não-invasiva, com resposta insatisfatória até a introdução de fármaco inotrópico (dobutamina $-5 \mu \mathrm{g} / \mathrm{kg} / \mathrm{min}$ ). As dosagens seqüenciais de troponina I foram de 2,2 e 0,8 (cerca de $6 \mathrm{~h}$ e $12 \mathrm{~h}$ após o evento, respectivamente); o ecocardiograma transtorácico demonstrou hipocinesia apical e o eletrocardiograma manteve-se semelhante ao de admissão, com distúrbio difuso da repolarização ventricular o que levou a equipe a considerar os possíveis diagnósticos de síndrome coronária aguda sem elevação do segmento ST ou disfunção miocárdica secundária à cardiotoxicidade por quimioterápico ou síndrome de reperfusão.

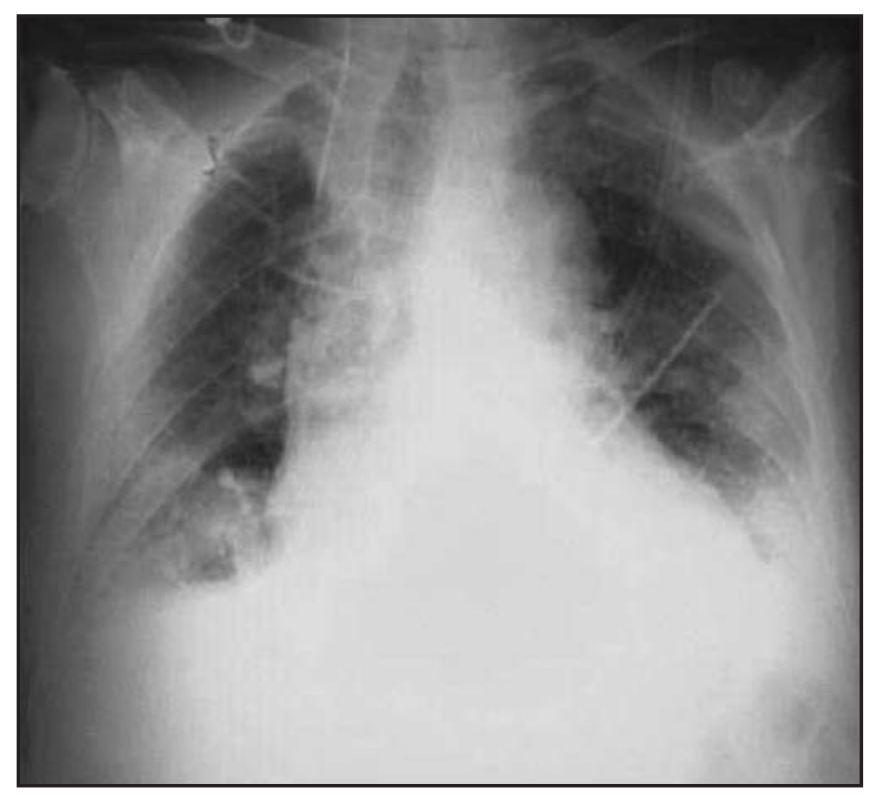

Figura 1 - Edema Agudo de Pulmão

A paciente permaneceu sob uso de fármaco inotrópico positivo (dobutamina) em doses entre 5 e $15 \mu \mathrm{g} / \mathrm{kg} / \mathrm{min}$ de acordo com parâmetros hemodinâmicos e de oxigenação (PVC, PA, FC, diurese e $\mathrm{SvO}_{2}$ ) e tratamento para disfunção miocárdica com furosemida, ramipril, espironolactona, enoxaparina e aspirina.

Recebeu alta da unidade de terapia intensiva para a enfermaria, com melhora do quadro, após 8 dias da admissão.

\section{DISCUSSÃO}

$\mathrm{Na}$ literatura, as opiniões sobre a técnica de perfusão isolada para o melanoma permanecem controversas, apesar de estudos com extensas casuísticas. Para ca- sos de melanoma inoperável esta técnica parece ser o tratamento de escolha, com diversos casos de erradicação completa da doença sob análise macroscópica, reduzindo o número de amputações e melhorando os sintomas de forma paliativa ${ }^{4,7,8}$.

O quimioterápico mais utilizado é o melfalan, quimicamente o p-di (2-cloroetil) amino-L-fenilalanina, um agente alquilante bifuncional (Figura 2).

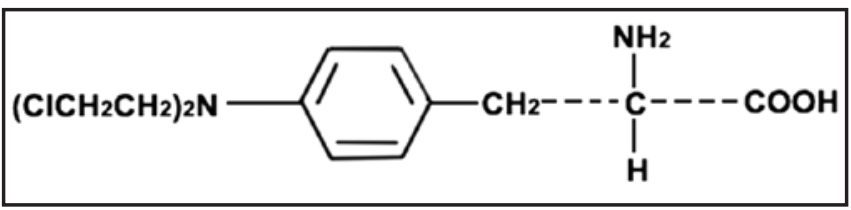

Figura 2 - Estrutura Química do Melfalan, p-di (2-cloroetil) amino-L-fenilalanina.

O fármaco carreia radicais alquilantes que atingem a célula tumoral e impedem a sua replicação. É pouco solúvel em água, mas dilui prontamente em álcool ou propilenoglicol. A administração por via venosa foi liberada em 1993, nos Estados Unidos, para uso no tratamento do mieloma múltiplo ${ }^{2,3}$.

O conhecimento sobre os efeitos adversos do tratamento não deve ser restrito ao cirurgião responsável pela execução e o seguimento do procedimento, mas também se estender à equipe clínica e/ou terapia intensiva para que a prevenção e o tratamento de eventuais disfunções orgânicas sejam precoces e efetivas, reduzindo o seu impacto na morbimortalidade após o tratamento imediato. A maior parte das publicações apontam apenas para efeitos da infusão por via venosa sistêmica, pouco se relatando efeitos da perfusão regional isolada ${ }^{4-6}$.

O efeito adverso mais comum é a depressão de medula óssea ocasionando leucopenia e trombocitopenia, em geral em torno de 14 a 21 dias, com recuperação em até 5 semanas. Convém citar, porém, a existência de relatos da ocorrência de lesão medular irreversível. Alguns casos de anemia hemolítica também foram descritos associados ao tratamento com melfalan ${ }^{4,5,7}$. Náuseas e vômitos podem ocorrer em até $30 \%$ dos pacientes. O manuseio de forma efetiva destes eventos deve levar em consideração o risco de possível desidratação acentuar o quadro de hipoperfusão tecidual, rabdomiólise e disfunção renal que estão associados a procedimento temporário ou permanente e comprometimento de circulação regional. A incidência de diarréia, vômitos e estomatite aumentam em pacientes que recebem altas doses do melfalan. Raras alterações 
hepáticas, sendo algumas com manifestações clínicas, semelhantes às descritas por toxicidade de diversos fármacos, podem ocorrer.

Raros casos de reações de hipersensibilidade $(2,4 \%)$, tais como urticária, edema, eritema cutâneo e anafilaxia foram relatados após a administração de doses iniciais ou subseqüentes, particularmente, após administração venosa e raramente associada à parada cardíaca. Ocasionalmente observaram-se eritemas maculopapulares e prurido. Não há relatos de que a perfusão regional possa provocar estes sintomas de forma disseminada, mas apenas in loco.

Não tem sido descrita a associação do melfalan a situações de cardiotoxicidade ${ }^{9}$, como déficit da contratilidade cardíaca, porém sua possível associação à fibrose endocárdica foi relatada por Sanz Manrique e col. ${ }^{7}$ em análise mais tardia, e também está relatada a chamada síndrome "choque séptico - like" (SIRS) com incidência em torno de $12 \%$ a $29 \%{ }^{10}$, com eventual disfunção miocárdica semelhante a disfunção miocárdica associada à sepse, o que pode ter acontecido ou corroborado para eventual disfunção e edema agudo pulmonar apresentado pela paciente.

Convém citar que as reações sistêmicas ao melfalan na perfusão isolada de membro são de ocorrência menor que $5 \%$, sendo muito mais freqüente quando se associa o fator de necrose tumoral ${ }^{11}$.

\section{CONCLUSÃO}

As manifestações sistêmicas adversas em intervenções terapêuticas em Oncologia, ainda que em perfu- são isolada de membro, podem acarretar riscos adicionais de morbidade dos pacientes, justificando a necessidade do conhecimento e cuidado adequado para manuseio e prevenção de eventos adversos pela equipe de terapia intensiva, bem como entender suas eventuais correlações com o tratamento instituído.

\section{REFERÊNCIAS}

01. Tsao H, Atkins MB, Sober AJ - Management of cutaneous melanoma. N Engl J Med, 2004;351:998-1012.

02. Krementz ET, Carter R, Sutherland CM et al - Regional chemotherapy for melanoma. A 35-year experience. Ann Surg, 1994;220:520-535.

03. Decian F, Balletto N, Camerini G et al - Hyperthermic-antiblastic isolation perfusion for advanced melanoma of the limbs. The technic, immediate results and a review of the literature. Minerva Chir, 1990;45:1227-1237.

04. ONS Clinical Practice Committee. Cancer Chemotherapy Guidelines and Recommendations for Practice. Pittsburgh, PA: Oncology Nursing Society; 1999:32-41.

05. Recommendations for the safe handling of parenteral antineoplastic drugs. Washington, DC: Division of Safety, Clinical Center Pharmacy Department and Cancer Nursing Services, National Institutes of Health; 1992. US Dept of Health and Human Services. Public Health Service Publication NIH 92-2621.

06. ASHP technical assistance bulletin on handling cytotoxic and hazardous drugs. Am J Hosp

07. Sanz Manrique N, Valcarce Perez J, Broto Escapa P et al - Enhancing factors in the cardiotoxicity of anthracyclines. An Esp Pediatr, 1990;32:11-14.

08. Ritchie DS, Seymour JF, Roberts AW et al - Acute left ventricular failure following melphalan and fludarabine conditioning. Bone Marrow Transplant, 2001;28(1):101-103.

09. Bitran JD. Doxorubicin cardiotoxicity and melphalan. Ann Intern Med, 1981;95(2):243-244.

10. Fraker DL, Alexander HR, Ross M - A phase III trial of isolated limb perfusion for extremity melanoma comparing melphalan alone versus melphalan plus tumor necrosis factor (TNF) plus interferon-gamma (IFN). Ann Surg Oncol, 2002;9:(Suppl):S8.

11. Cornett WR, McCall LM, Petersen RP - et al - Randomized multicenter trial of hyperthermic isolated limb perfusion with melphalan alone compared with melphalan plus tumor necrosis factor: American College of Surgeons Oncology Group Trial Z0020. J Clin Oncol, 2006;24:4196-4201. 